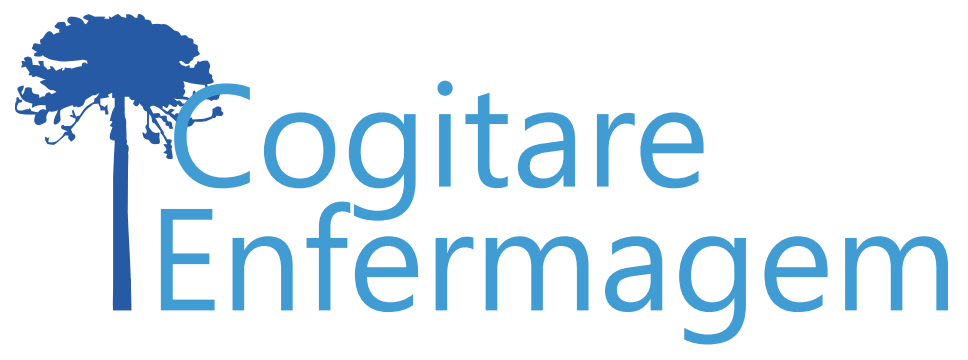

\title{
CONHECIMENTO E ATITUDES DE PROFISSIONAIS DE ENFERMAGEM DE UM HOSPITAL PAULISTA FRENTE ÀS PRECAUÇÕES PADRÃO
}

Edvan Adalberto Dias dos Passos', Maria Helena Palucci Marziale²

\section{RESUMO}

Objetivo: analisar conhecimentos e atitudes de profissionais de enfermagem sobre adesão às Precauções Padrão.

Método: estudo descritivo. Amostra composta por 165 profissionais da enfermagem de um hospital paulista. Dados coletados pelos Questionnaires for knowledge and Compliance with Standard Precaution - versão em português e analisados por estatística descritiva com cálculo de frequência. Resultados: a maioria dos profissionais $(98,2 \%)$ conhece as Precauções Padrão, porém 19,4\% responderam corretamente todas as questões formuladas. Atitudes adequadas foram identificadas em relação ao descarte de materiais perfurocortantes $(99,4 \%)$, uso de luvas $(91 \%)$ e higienização das mãos $(89,8 \%)$. Comportamento de risco foi identificado pelo não uso de máscaras $(64,6 \%)$ e óculos de proteção (54,9\%).

Conclusão: a maioria dos profissionais conhece as Precauções Padrão, porém a atitude de adesão é parcial. Esse estudo contribui para o planejamento de ações de segurança no trabalho e a elaboração de novas pesquisas sobre comportamentos de risco de profissionais de enfermagem.

DESCRITORES: Acidentes de Trabalho; Enfermagem; Saúde do Trabalhador; Exposição Ocupacional; Precauções Universais.

COMO REFERENCIAR ESTE ARTIGO:

Passos EAD dos, Marziale MHP. Conhecimento e atitudes de profissionais de enfermagem de um hospital paulista frente às precauções padrão. Cogitare enferm. [Internet]. 2020 [acesso em "colocar data de acesso, dia, mês abreviado e ano"]; 25. Disponível em: http://dx.doi.org/10.5380/ce.v25i0.66744.

\section{(c) (1)}

Este obra está licenciado com uma Licença Creative Commons Atribuição 4.0 Internacional. 


\title{
KNOWLEDGE AND ATTITUDES OF NURSING PROFESSIONALS AT A HOSPITAL IN THE BRAZILIAN STATE OF SÃO PAULO REGARDING STANDARD PRECAUTIONS
}

\begin{abstract}
Objective: to analyze the knowledge and attitudes of nursing professionals regarding adherence to standard precautions.

Methods: descriptive study. The sample was made up of 165 nursing professionals who worked at a hospital in the Brazilian state of São Paulo. Data were collected by applying the Portuguese version of the Questionnaires for Knowledge and Compliance with Standard Precaution and analyzed by using descriptive statistics with frequency calculation.

Results: most of the professionals (98.2\%) knew standard precautions, but $19.4 \%$ answered all the questions correctly. Adequate attitudes were identified concerning disposal of sharps (99.4\%), use of gloves (91\%), and hand hygiene (89.8\%). Risk behavior was detected by the nonuse of masks (64.6\%) and goggles (54.9\%).

Conclusion: most of the analyzed professionals knew standard precautions, but their adherence attitude was partial. The present study contributes to planning safety actions at work and designing new studies on risk behaviors in nursing professionals.
\end{abstract}

DESCRIPTORS: Occupational Accidents; Nursing; Occupational Health; Occupational Exposure; Universal Precautions.

\section{CONOCIMIENTO Y ACTITUDES DE PROFESIONALES ENFERMEROS DE HOSPITAL PAULISTA RESPECTO DE LAS PRECAUCIONES ESTANDAR}

\begin{abstract}
RESUMEN
Objetivo: Analizar conocimientos y actitudes de profesionales enfermeros sobre adhesión a las precauciones estándar.

Método: Estudio descriptivo. Muestra integrada por 165 profesionales enfermeros de un hospital paulista. Datos recolectados mediante Questionnaires for knowledge and Compliance with Standard Precaution - versión en portugués, analizados por estadística descriptiva con cálculo de frecuencia.

Resultados: La mayoría de los profesionales (98,2\%) conoce las precauciones estándar, aunque solo el 19,4\% respondió correctamente todas las preguntas. Fueron identificadas actitudes adecuadas respecto a descarte de materiales punzocortantes $(99,4 \%)$, uso de guantes $(91 \%)$ e higiene de manos $(89,8 \%)$. Se identificó comportamiento riesgoso por no utilización de barbijos $(64,6 \%)$ y protectores oculares $(54,9 \%)$.

Conclusión: La mayoría de los profesionales conoce las precauciones estándar, aunque muestran una adhesión parcial. El estudio contribuye a planificar acciones de seguridad laboral y a elaborar nuevas investigaciones sobre comportamientos de riesgo de profesionales enfermeros.
\end{abstract}

DESCRIPTORES: Accidentes de Trabajo; Enfermería; Salud Laboral; Exposición Profesional; Precauciones Universales. 
O ambiente de trabalho hospitalar oferece grandes riscos ocupacionais aos profissionais que nele estão inseridos, podendo lhes causar acidentes e adoecimentos. Tais riscos estão relacionados à organização e dinâmica do ambiente de trabalho, à qualidade e quantidade de materiais dispostos para uso, às características individuais de cada profissional, além do intenso ritmo de trabalho ${ }^{(1,2)}$.

Dentre os riscos de injúria a que um profissional está sujeito dentro do ambiente hospitalar, o mais comum são os com agentes biológicos, por estarem relacionados à transmissão de doenças e insalubridade pelo contato direto do profissional de enfermagem com os pacientes. Diante dos riscos biológicos, as doenças mais preocupantes são aquelas causadas pelos vírus da Adquired Immunity Deficiency Şyndrome-AIDS (HIV), Hepatite C e $B\left(\mathrm{HCV}\right.$ e HBV) ${ }^{(1-3)}$.

A enfermagem integra uma categoria profissional que está exposta à ocorrência de acidentes de trabalho com material biológico (ATMB), por executar atividades em contato constante e direto com o paciente, e manipular materiais potencialmente contaminados por agentes infecciosos e materiais perfurocortantes como agulhas, vidraria e lâminas.

Considerando as efetivas possibilidades de transmissão de doenças infectocontagiosas por ocasião dos acidentes de trabalho, medidas preventivas foram internacionalmente recomendadas para diminuir o referido risco ocupacional, denominadas Precauções Padrão (PP) do Centro de Controle e Prevenção de Doenças (CDC) ${ }^{(3)}$. Trata-se de medidas que visam minimizar riscos e o uso adequado de equipamentos de proteção individuais (EPIs). Estes últimos são a principal barreira de proteção para o profissional na prevenção de acidentes, porém há resistência por parte dos trabalhadores na sua adesão, e o uso incorreto dos EPls aumenta a chance de exposição ao material biológico ${ }^{(2,4)}$.

Embora seja eficaz a adesão das PP na prevenção à pré-exposição a material biológico, ainda há baixa adesão às PP por parte dos profissionais da saúde ${ }^{(5,6)}$. A literatura traz que a baixa adesão pode estar relacionada ao desconforto, incômodo, descuido, esquecimento, falta de hábito, inadequação dos equipamentos, falta de material(7).

Em função disto, compreender e estudar o conhecimento sobre as PP e atitudes dos profissionais de saúde frente ao risco é de fundamental importância para que se possa levantar estratégias e estudos que proporcionem melhora nas condições de trabalho de profissionais da saúde. Assim, o objetivo deste estudo foi analisar o conhecimento e atitudes dos profissionais de enfermagem sobre a adesão às Precauções Padrão.

\section{MÉTODO}

Estudo descritivo de abordagem quantitativa. Os dados foram coletados em um hospital de ensino de Ribeirão Preto-SP, no período de janeiro a dezembro de 2016. A amostra foi composta por 165 profissionais de enfermagem, sendo 39 enfermeiros, 60 técnicos e 66 auxiliares. Os critérios de inclusão foram todos os profissionais que sofreram acidentes de trabalho com exposição a material biológico registrados no Serviço de Segurança e Medicina do Trabalho (SُESMT) do hospital estudado e na Rede de Prevenção de Acidentes de Trabalho - REPAT/USP(8).

Os dados foram coletados com uso dos Questionnaires for knowledge and compliance with standard precaution, construído e validado por $L^{\left({ }^{(9)}\right.}$ e adaptado para a realidade brasileira por Valim(10). $\mathrm{O}$ instrumento é composto por questionário para caracterização sociodemográfica, questionário de adesão às precauções padrão e questionário de conhecimento sobre as PP. Os questionários são autoaplicáveis e impressos em folhas de papel e foram entregues aos profissionais de enfermagem. Foi feito um levantamento junto 
ao SESMT dos profissionais de enfermagem que sofreram ATMB e a unidade do hospital em que trabalhavam, esses profissionais foram contatados pelo pesquisador, sendo-lhes entregue os instrumentos de coleta de dados para que eles os preenchessem e agendada data para o seu recolhimento.

O questionário de caracterização sociodemográfica e ocupacional contempla as seguintes variáveis: sexo, setor de trabalho, data de nascimento, estado civil, grau de escolaridade, local de atuação profissional, tempo de experiência profissional, vacinação contra hepatite B e conhecimento da presença do anticorpo anti-HBS, participação e desejo de participação em treinamento sobre as medidas de PP, ocorrência de acidente de trabalho com material biológico, notificação de acidente com material biológico, realização da troca de recipiente de descarte material perfurocortante e foi validado quanto ao conteúdo. Já o questionário de conhecimento sobre as PP é composto por 20 questões referentes ao conceito básico de PP e aos conteúdos e exigências práticas com relação às $\mathrm{PP}$, tais como a necessidade de higienização das mãos, uso de EPI, práticas seguras no manuseio de material perfurocortante e prevenção de infecção.

As respostas possíveis são: "sim", "não" ou "desconhecido". A cada resposta correta é somado o valor de um ponto, enquanto às respostas dadas como incorretas e desconhecidas nada é somado (0 pontos). A maior pontuação possível são 20 pontos e, quanto maior a pontuação, maior o conhecimento do indivíduo sobre as PP. O processo de validação desse instrumento obteve validade de conteúdo de 0,98 ; confiabilidade por meio do teste-reteste de 0,86 e consistência interna de $0,92^{(10)}$.

O questionário de adesão às PP é composto por 20 questões relacionadas à adesão de profissional de saúde às $\mathrm{PP}$ e foi desenvolvido em formato de escala do tipo Likert, variando de 0 a 4 pontos. A cada resposta obtida como "sempre" são somados 4 pontos; "frequentemente" somam-se 3 pontos; "às vezes" somam-se 2 pontos; "raramente" somase 1 ponto e "nunca" nada se soma (0 pontos), com exceção da questão de número 20 (item inverso). O intervalo de pontuação varia de 0 a 80 pontos. Quanto maior a pontuação, mais o indivíduo segue as medidas de PP. O processo de validação do referido questionário obteve validade pelo conteúdo de 0,98; confiabilidade por meio do teste-reteste de 0,87 e consistência interna de 0,93 obtida pelo alfa de Cronbach $^{(10)}$. Os resultados da pesquisa são apresentados por gráficos e tabelas.

O estudo faz parte da pesquisa intitulada "Rede de Prevenção Acidentes de Trabalho - REPAT/USP: adesão às medidas preventivas primárias a exposição ocupacional a material biológico", que foi aprovada pelo Comitê de Ética em Pesquisa, sob parecer 053/2015, registro CAAE: 43032815.0.0000.5396. Ao longo da pesquisa e divulgação dos dados, garantimos o sigilo e anonimato de cada participante.

\section{RESULTADOS}

Dos 165 profissionais participantes do estudo, 39 (23,6\%) eram enfermeiros, 60 $(36,4 \%)$ técnicos de enfermagem e $66(40 \%)$ auxiliares de enfermagem. Dos participantes, $125(75,8 \%)$ eram do sexo feminino, com idade média de 40,4 anos (com variação entre 24,9 a 65,9 anos).

A Figura 1 mostra a frequência de notificações de acidentes de trabalho de acordo com a distribuição da quantidade de acidentes. Nota-se que $61,8 \%$ dos participantes da pesquisa não notificaram os acidentes de trabalho que sofreram, enquanto $11 \%$ relataram terem sofrido um acidente. $0,6 \%$ dos componentes da pesquisa relataram 8 ATBM. 


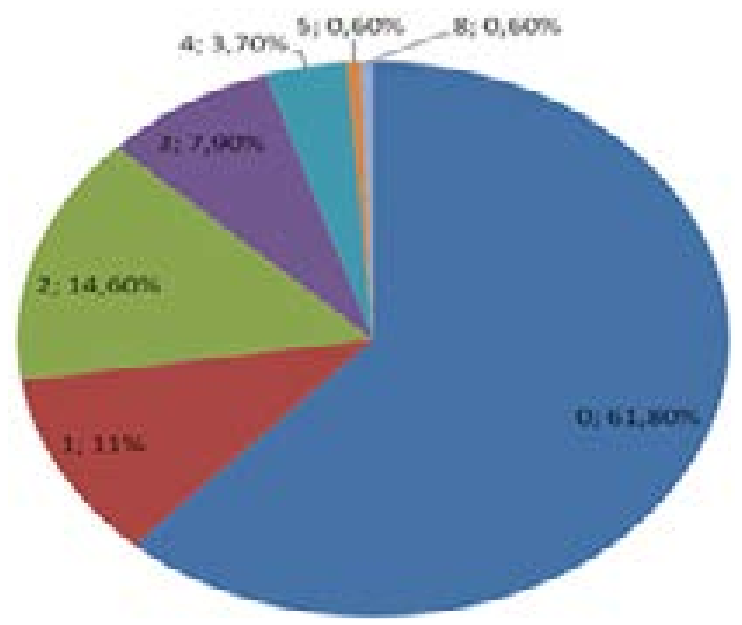

Figura 1 - Distribuição de acidentes de trabalho com exposição a material biológico entre profissionais de enfermagem de acordo com a frequência numérica percentual. Ribeirão Preto, SP, Brasil, 2016

A Tabela 1 apresenta os dados do conhecimento dos profissionais participantes em relação a conceitos básicos sobre as precauções padrão. Dos participantes, $161(98,2 \%)$ relataram ter conhecimento sobre o que são as PP, enquanto três (1,8\%) disseram não ter conhecimento. Sobre a proibição de dobrar, entortar ou realizar encape ativo das agulhas, 141 (86\%) profissionais responderam assertivamente à proibição. enquanto $22(13,4 \%)$ relataram não conhecer o impedimento em realizar os referidos atos. 132 participantes $(80,5 \%)$ disseram ser necessário adotar apenas as PP ao prestar cuidado a pacientes com sífilis, hepatites, enquanto 25 (15,2\%) disseram não ser suficientes.

Tabela 1 - Conhecimento de profissionais de enfermagem sobre as Precauções Padrão. Ribeirão Preto, SP, Brasil, 2016

MEDIDAS DE PRECAUÇÕES-PADRÃO (PP)

\begin{tabular}{|c|c|c|c|c|}
\hline Situação & Sim & $\begin{array}{l}\text { Não } \\
\text { n/\% }\end{array}$ & $\begin{array}{c}\text { Desconhecido } \\
\text { n/\% }\end{array}$ & $\begin{array}{l}\text { Dados } \\
\text { Omissos } \\
\text { n/\% }\end{array}$ \\
\hline - Conhece o que são Precauções-Padrão. & $\begin{array}{c}161 \\
(98,20 \%)\end{array}$ & $\begin{array}{c}0 \\
(0 \%)\end{array}$ & $\begin{array}{c}3 \\
(1,80 \%)\end{array}$ & 1 \\
\hline $\begin{array}{l}\text { - Conhece que é proibido dobrar, entortar ou } \\
\text { realizar o encape ativo de agulhas. Quando } \\
\text { necessário realizar o encape passivo. }\end{array}$ & $\begin{array}{c}141 \\
(86 \%)\end{array}$ & $\begin{array}{c}22 \\
(13,40 \%)\end{array}$ & $\begin{array}{c}1 \\
(0,60 \%)\end{array}$ & 1 \\
\hline $\begin{array}{l}\text { - Conhece que ao prestar cuidados à pacientes } \\
\text { com hepatite C ou sífilis é necessário adotar } \\
\text { apenas as PP. }\end{array}$ & $\begin{array}{c}132 \\
(80,50 \%)\end{array}$ & $\begin{array}{c}25 \\
(15,20 \%)\end{array}$ & $\begin{array}{c}7 \\
(4,30 \%)\end{array}$ & 1 \\
\hline $\begin{array}{l}\text { - Conhece que ao prestar cuidados a pacientes } \\
\text { com tuberculose ou varicela, é necessário adotar } \\
\text { as PP, além das precauções por gotículas. }\end{array}$ & $\begin{array}{c}140 \\
(85,40 \%)\end{array}$ & $\begin{array}{c}24 \\
(14,60 \%)\end{array}$ & - & 1 \\
\hline
\end{tabular}


A Figura 2 mostra a utilização de EPI recomendados nas PP. Observa-se que 106 $(64,6 \%)$ profissionais relataram sempre fazer uso de máscaras de proteção, enquanto três $(1,8 \%)$ e um $(0,6 \%)$ dos participantes disseram raramente ou nunca aderir a tal EPI. Já em relação aos óculos de proteção, 90 (54,9\%) disseram sempre fazer uso do EPI, 40 (24,4\%) frequentemente, $27(16,5 \%)$ às vezes e sete $(4,3 \%)$ raramente. O EPI avental sempre foi aderido por $97(59,1 \%)$ dos participantes, $43(26,2 \%)$ aderiram frequentemente, $19(11,6 \%)$ às vezes e cinco $(3 \%)$ nunca.

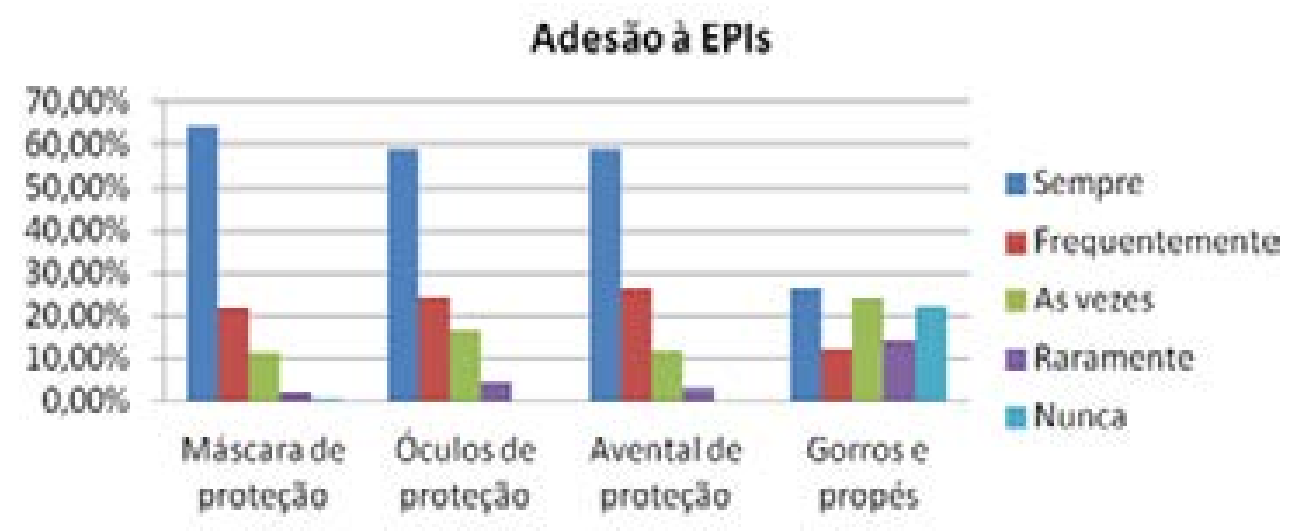

Figura 2 - Adesão às Precauções Padrão segundo uso de Equipamentos de Proteção Individual durante procedimentos e após acidente de trabalho. Ribeirão Preto, SP, Brasil, 2016

As Figuras 3 e 4 mostram a intenção dos profissionais de enfermagem em aderir às PP segundo as variáveis higienização das mãos e uso de luvas. Sobre o ato de higienizar as mãos, $158(96,3 \%)$ trabalhadores relataram realizá-lo após contato com material potencialmente contaminado, e apenas 146 (89\%) sempre higienizavam as mãos após retirar as luvas. E $138(84,1 \%)$ profissionais relataram realizar o procedimento entre o cuidado a diferentes pacientes.

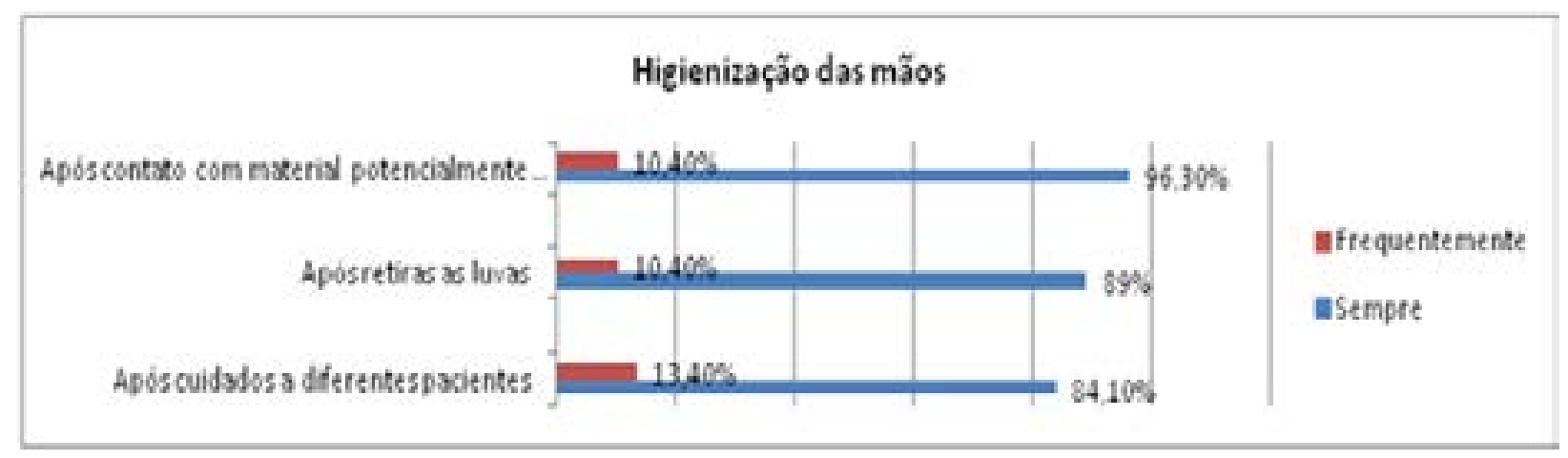

Figura 3 - Distribuição da frequência da realização de higienização das mãos por profissionais de Enfermagem. Ribeirão Preto, SP, Brasil, 2016 


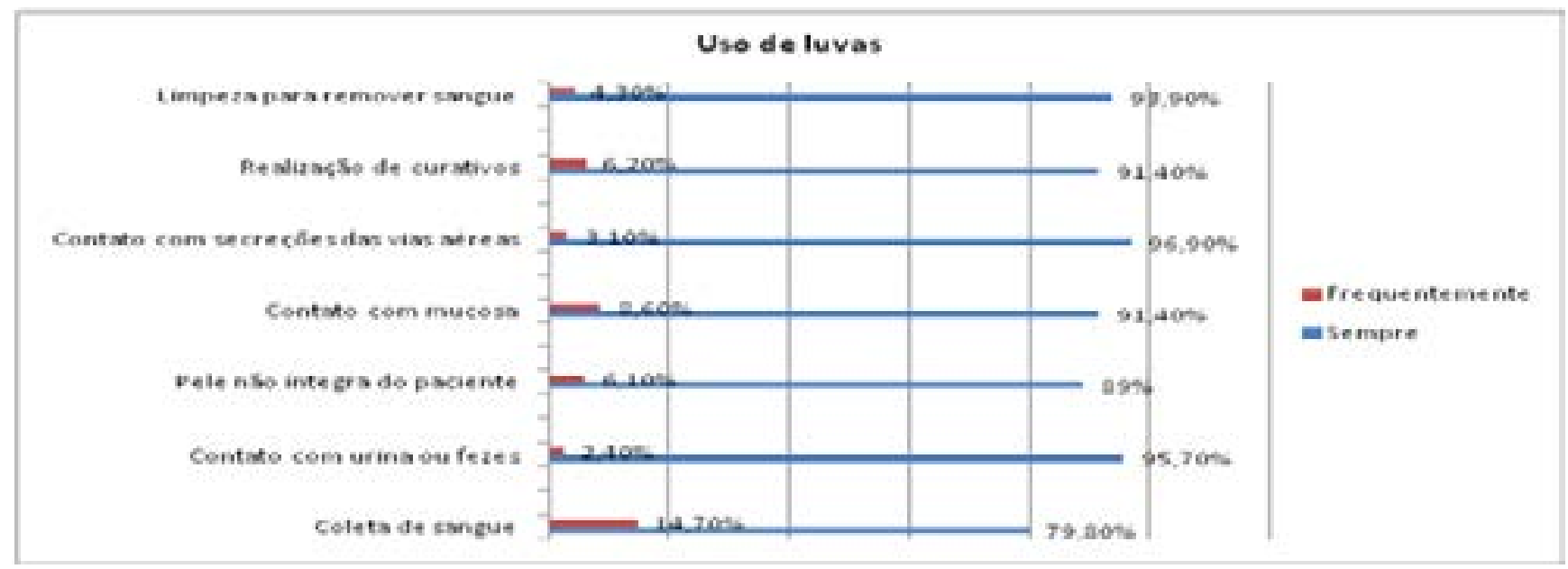

Figura 4 - Distribuição da frequência do uso de luvas por profissionais de enfermagem. Ribeirão Preto, SP, Brasil, 2016

O uso constante de luvas para limpar ou remover sangue foi relatado por 153 (93,9\%) trabalhadores, enquanto sete $(4,3 \%)$ disseram aderir frequentemente ao EPI para tal situação. Para a realização de curativos, $148(91,4 \%)$ alegaram sempre fazer o uso de luvas de procedimento e $10(6,2 \%)$ frequentemente. Dos participantes, 145 (89\%) profissionais responderam sempre fazer uso de luvas para contato com pele não íntegra, já $10(6,1 \%)$ disseram frequentemente aderir às luvas. Para a coleta de sangue, $130(79,8 \%)$ profissionais sempre aderem às luvas e 24 (14,7\%) declararam usar frequentemente luvas de proteção.

\section{DISCUSSÃO}

Os trabalhadores de enfermagem pertencentes à amostra estudada eram majoritariamente do sexo feminino, com média de idade igual a 40 anos. $O$ tempo de trabalho na Enfermagem variou entre 2 meses a 41 anos, o que mostra que o tempo de serviço não é um fator impeditivo para a ocorrência de acidentes de trabalho com exposição a material biológico. No entanto, estudo ${ }^{(11)}$ realizado com profissionais de enfermagem em um hospital universitário do interior paulista revela que, quanto maior a experiência do profissional, há maior tendência em aderir às medidas de precauçõespadrão, destacando a higienização das mãos. No entanto, outro estudo ${ }^{(5)}$, realizado com 590 profissionais de enfermagem de uma UTI em um hospital de ensino Paulista, identificou que a maior experiência dos profissionais pode levar à não adesão às precauções padrão, por se sentirem mais seguros ao prestar cuidados e dispensarem o uso de EPls. Cabe destaque que $10(6,1 \%)$ profissionais que sofreram acidentes de trabalho tinham o título de mestrado e $19(11,5 \%)$ de especialização.

Os resultados revelaram que, apesar de conhecerem os conceitos relacionados às PP, muitos trabalhadores desconhecem as informações necessárias em sua totalidade, necessitando de imediata atualização de informações, embora a maioria dos trabalhadores (89\%) tenha participado de programas educativos. O treinamento e atualização de informações foi identificado em estudo realizado com profissionais de enfermagem como um indicador de redutor do risco de exposição e por aumentar a adesão às PP(11).

Constatou-se que 161 (98,2\%) participantes responderam conhecer o que são as medidas de precauções-padrão, no entanto, alguns conceitos errôneos e equivocados foram identificados em relação à higienização das mãos durante a prestação de cuidados a pacientes diferentes, quanto à s situações em que uso de óculos e gorros são necessários, 
e quanto ao encape ativo de agulhas, e sobre quais as recomendações adequadas para assistir pacientes com doenças venéreas e com tuberculose. Outros estudos ${ }^{(12-14)}$ também evidenciaram resultados semelhantes de que alguns profissionais de enfermagem não possuem conhecimento adequado quanto ao uso correto dos EPIs e que contínua atenção deve ser direcionada à educação continuada nos serviços de saúde.

O reduzido uso de alguns EPIs, principalmente máscara e óculos de proteção, pode estar relacionado à falta de informação dos profissionais sobre a maneira correta de utilizálos, mas também a atitudes comportamentais que merecem atenção dos gestores dos serviços onde comportamentos de riscos devem ser trabalhados e coibidos.

Uma revisão integrativa(2) e um estudo ${ }^{(15)}$ realizado na clínica médica de um hospital de ensino do Triângulo Mineiro (MG) constataram que também em outros hospitais, embora a adesão às PP seja a principal estratégia para evitar principalmente infecções cruzadas e promover segurança aos pacientes e seus familiares e aos profissionais de saúde, a adesão às PP pelos profissionais de saúde ainda se encontra abaixo do esperado e desejável.

Todos os profissionais devem realizar a higienização das mãos, antes e após a prestação de cuidado a cada paciente, mesmo quando há uso luvas de proteção e ao manipular material biológico para a sua própria segurança e do paciente ${ }^{(3)}$, uma vez que a inadequada higienização das mãos pelos profissionais de saúde entre o atendimento de pacientes é comprovadamente uma fonte de contaminação. No entanto, houve baixa adesão por parte dos profissionais em relação a esse procedimento, conforme recomendado pelas PP: $138(84,1 \%)$ participantes declararam realizar a higienização das mãos entre a prestação de cuidados a pacientes diferentes, 146 (89\%) responderam higienizar as mãos após retirar as luvas e 158 (96,3\%) após contato com material biológico. Esse problema também foi identificado em estudos realizados em outros hospitais ${ }^{(11-16)}$.

Aluva foi o EPI mais utilizado pelos trabalhadores deste estudo, durante procedimentos de coleta de sangue, em situações em que há possibilidade de contato com urina e fezes, secreções, realizações de curativos, limpeza e contato com sangue. Outros estudos também identificaram a luva como principal EPI adotado pelos profissionais de enfermagem na prestação do cuidado(11-13,16).

Houve baixa adesão por parte dos participantes em relação à utilização de máscaras $106(64,6 \%)$, óculos $90(54,9 \%)$ e aventais de proteção $97(59,1 \%)$. Isso também foi observado em outras pesquisas ${ }^{(11,16)}$. Nesses estudos, os profissionais de enfermagem que não usam óculos de proteção referem como motivo que os óculos embaçam e dificultam a visão e que tem dúvidas sobre quando devem utilizá-los ${ }^{(11)}$, esse mesmo motivo foi apresentado quanto à baixa adesão ao uso de máscaras de proteção, somada ao incômodo para respirar.

Os óculos de proteção devem ser utilizados para proteção dos olhos e face durante procedimentos em que há possibilidade de respingos ou contra impacto de objetos ${ }^{(17)}$. $O$ uso correto da máscara de proteção é para proteção das vias aéreas superiores e mucosa oral, e deve também ser utilizada de acordo com o material a ser manipulado, por exemplo, manipulação de agentes biológicos patogênicos com alta probabilidade de geração de aerossóis $^{(17)}$.

Muitos trabalhadores declararam realizar o encape ativo de agulhas ou realizar o encape passivo com apenas uma das mãos, mesmo sendo esta conduta proibida pela legislação brasileira NR32(18). Essa prática não é recomendável internacionalmente, porém ainda é observada a sua realização ${ }^{(11,12)}$ entre profissionais de enfermagem que continuam assumindo esse comportamento de risco. Essa prática aumenta consideravelmente o risco do trabalhador se acidentar, devido ao risco de a agulha romper a capa protetora e perfurar os dedos de quem a está manipulando. A literatura evidencia que, ao realizar o encape ativo, as chances do profissional se acidentar aumentam em até 25 vezes $^{(19)}$. Em muitos países, é exigido legalmente que as instituições de saúde disponibilizem aos profissionais de saúde agulhas com dispositivos de segurança, e estudos comprovam que tais dispositivos reduzem a ocorrência de acidentes perfurantes com agulhas e dispositivos intravenosos ${ }^{(20)}$. 
Dentre os motivos de baixa adesão às PP apresentados em outras pesquisas, estão as inadequadas condições de trabalho oferecidas, a não disponibilização pelas instituições empregadoras de materiais e equipamentos de segurança adequados, falta de programas de educação continuada, sobrecarga de trabalho, e falta de conhecimento dos trabalhadores de como manusear EPIs, a exemplo das seringas com agulhas retráteis ${ }^{(11,12,20)}$.

Por fim, a conscientização do trabalhador sobre a importância em aderir às PP, garantindo que o cuidado seja mais seguro ao profissional e paciente, também pode ser um fator determinante para a adesão às $\mathrm{PP}^{(20)}$.

Destaca-se ainda que comportamentos de risco à segurança no trabalho de enfermagem devem ser coibidos e podem ser modificados com a adoção de práticas educativas e pelo processo de formação de profissionais de enfermagem.

As limitações do estudo são relacionadas à coleta dos dados, considerando quatro principais pontos: localizar os trabalhadores acidentados; interesse em participar da pesquisa; entregar no prazo combinado; e número de questionários respondidos por completo.

A localização dos participantes, após feito o levantamento dos profissionais acidentados junto ao SESMT, era dificultada pelo fato de que muitos dos profissionais não trabalhavam mais no local em que constava na indicação do SESMT, tendo que ser realizada uma busca ativa em todos os andares do hospital estudado, que era um total de 12 andares com duas alas cada um. Outro ponto que dificultou o estudo era a aceitação dos trabalhadores em participar da pesquisa. Estes alegavam falta de tempo, pela sobrecarga de trabalho, ter de participar de diversos outros estudos, por trabalharem em um hospital escola. A falta de motivação dos trabalhadores participantes, o tempo curto, para quem optava em responder o questionário no local de trabalho, dificultava o preenchimento do instrumento, o que resultou em questionários preenchidos de forma errada, tendo que ser excluídos do estudo.

\section{CONCLUSÃO}

Mesmo sendo internacionalmente recomendadas, a adesão às PP foi parcial pelos profissionais de enfermagem devido a fatores individuais e organizacionais. Destaca-se a necessidade de ampliar o oferecimento de informações sobre as medidas necessárias para prevenção de infecção cruzada e quando e como EP̉ como óculos e máscaras de proteção devem ser usados.

Considera-se necessário intensificar a oferta de programas de educação permanente para os trabalhadores de Enfermagem e aplicar estratégias que efetivamente estimulem a mudança dos comportamentos de risco para comportamentos seguros de trabalho. Recomenda-se a inclusão nas ações preventivas a prática educativa e a participação de pessoas consideradas líderes pelo grupo de trabalhadores, para servirem como bons exemplos e auxiliar a maior adesão às PP. Da mesma forma, promover discussões com a equipe de enfermagem sobre a postura frente ao risco e adesão às PP, para assegurar comportamentos seguros, que minimizem a ocorrência de acidentes de trabalho e o adoecimento pelo trabalho.

O estudo agrega conhecimento científico para a área de Enfermagem e Saúde do Trabalhador, por apresentar dados relacionados a lacunas de informações dos trabalhadores de enfermagem, as quais devem ser contempladas desde a formação e nas práticas de educação em serviço. Os dados indicam também a necessidade de um olhar mais amplo aos fatores que envolvem a situação de trabalho de Enfermagem, buscando a prevenção dos acidentes e a melhoria das condições de trabalho. 
Ao Conselho Nacional de Desenvolvimento Científico e Tecnológico (CNPq), processo $n^{\circ} 103328 / 2015-7$, pelo financiamento do projeto de pesquisa.

\section{REFERÊNCIAS}

1. Ribeiro AS, Gabatz RIB, Neves, Padoin SM de M. Caracterização de acidente com material perfurocortante e a percepção da equipe de enfermagem. Cogitare enferm. [Internet]. 2009 [acesso em 21 jul 2017]; 14(4). Disponível em: http://www.revenf.bvs.br/scielo.php?script=sci arttext\&pid=S1414-

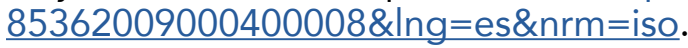

2. Porto JS, Marziale MHP. Motivos e consequências da baixa adesão às precauções padrão pela equipe de enfermagem. Rev. Gaúcha Enferm. [Internet]. 2016 [acesso em 04 out 2017]; 37(2). Disponível em: http://dx.doi.org/10.1590/1983-1447.2016.02.57395.

3. Centers For Disease Control And Prevention (CDC). US. Public health Service Guidelines for the managementof occupational exposures to HBV HCV, and HIV and rRecomendations for postexposure prophylaxis. MMWR Recomendações e relatórios. [Internet]. 2001. [acesso em 04 out 2017]; 50(11). Disponível em: https://www.cdc.gov/mmwr/preview/mmwrhtml/rr5011a1.htm.

4. Marziale MHP, Santos HEC dos, Cenzi CM, Rocha FLR, Trovó MEM. Consequências da exposição ocupacional a material biológico entre trabalhadores de um hospital universitário. Esc. Anna Nery. [Internet]. 2014 [acesso em 04 out 2017]; 18(1). Disponível em: http://dx.doi.org/10.5935/14148145.20140002.

5. Malaguti-Toffano SE, Canini SEM da S, Reis RK, Pereira FMV, Felix AM da S, Ribeiro PHV, et al. Adesão às precauções-padrão entre profissionais da enfermagem expostos a material biológico. Rev. Eletr. Enf. [Internet]. 2015 [acesso em 05 out 2017]; 17(1). Disponível em: http://dx.doi.org/10.5216/ree.v17i1.29269.

6. Valim MD, Marziale MHP. Avaliação da exposição ocupacional a material biológico em serviços de saúde. Texto contexto-enferm. [Internet]. 2011 [acesso em 04 out 2017]; 20(spe). Disponível em: http:// dx.doi.org/10.1590/S0104-07072011000500018.

7. Neves HCC, Souza ACS e, Medeiros M, Munari DB, Ribeiro LCM, Tipple AFV. Segurança dos trabalhadores de enfermagem e fatores determinantes para adesão aos equipamentos de proteção individual. Rev. Latino-Am. Enfermagem. [Internet]. 2011 [acesso em 21 jul 2017]; 19(2). Disponível em: http://www.scielo.br/pdf/rlae/v19n2/pt 18.

8. Rede de Prevenção de Acidentes de Trabalho com Exposição a Material Biológico em Hospitais Universitários do Brasil (REPAT) [Internet]. São Paulo: REPAT [acesso em 10 out 2017]. Disponível em: http://repat.eerp.usp.br.

9. Luo Y, He GP, Zhou JW, Luo Y. Factor's impacting compliance with standard precautions in nursing, China. Int. J. Infect. Dis. [Internet]. 2010 [acesso em 21 jul 2017]; 14(12). Disponível em: https://doi. org/10.1016/j.ijid.2009.03.037.

10. Valim MD, Marziale MHP, Hayashida M, Richart-Martínez M. Ocorrência de acidentes de trabalho com material biológico potencialmente contaminado em enfermeiros. Acta Paul. Enferm. [Internet]. 2014 [acesso em 04 out 2017]; 27(3). Disponível em: http://dx.doi.org/10.1590/1982-0194201400047.

11. Pereira FMV, Malaguti-Toffano SE, Silva AM da, Canini SEM da S, Gir E. Adesão às precauções-padrão pelos profissionais de enfermagem que atuam em terapia intensiva em um hospital universitário. Rev. Esc. Enferm. USP. [Internet]. 2013 [acesso em 04 out 2017]; 47(3). Disponível em: http://dx.doi.org/10.1590/ S0080-623420130000300023.

12. Lima RJV, Tourinho BCM de S, Costa D de S, Almeida DMPF de, Tapety FI, Landim-Almeida CAP, 
et al. Agentes biológicos e equipamentos de proteção individual e coletiva: conhecimento e utilização entre profissionais. Rev. Pre. Infec. e Saúde. [Internet]. 2017 [acesso em 05 out 2017]; 3(1). Disponível em: https://doi.org/10.26694/repis.v3i3.6684.

13. Ilapa-Rodriguez EO, Silva GG da, Lopes Neto D, Campos MP de A, Mattos MCT de, Otero LM. Medidas para la adhesión a las recomendaciones de bioseguridad para el equipo de enfermería. Enf. Global [Internet]. 2017 [acesso em 13 fev 2019]; 17(1). Disponível em: https://doi.org/10.6018/ eglobal.17.1.276931.

14. Valim MD, Morais RB de; Marziale MHP. Instrumentos y factores impactantes en el conocimiento de medidas de precaución estándar entre trabajadores de la salud. Enferm Global. [Internet]. 2016 [acesso em 04 out 2017]; 15(41). Disponível em: http://scielo.isciii.es/scielo.php?script=sci_arttext\&pid=S1695$\underline{61412016000100014 \& \operatorname{lng}=\text { es. }}$.

15. Ferreira LA, Peixoto $C$ de $A$, Paiva $L$, Silva $Q C G$ da, Rezende MP, Barbosa $M H$. Adesão às precauções padrão em um hospital de ensino. Rev. bras. enferm. [Internet]. 2017 [acesso em 04 out 2017]; 70(1). Disponível em: http://dx.doi.org/10.1590/0034-7167-2016-0138.

16. Piai-Morais TH, Orlandi $F$ de $S$, Figueiredo RM de. Fatores que influenciam a adesão às precauções-padrão entre profissionais de enfermagem em hospital psiquiátrico. Rev. Esc. Enferm. USP. [Internet]. 2015 [acesso em 04 out 2017]; 49(3). Disponível em: http://dx.doi.org/10.1590/S0080623420150000300016.

17. Pimentel BJ, Santana CST de, Araújo DC de S, Silva ET da, Bomfim IQM, Faé J, et al. Manual de biossegurança enfermagem. Maceió: Centro Universitário CESMAC; 2015 [acesso em 04 out 2017]. Disponível em: https://cesmac.edu.br/admin/wp-content/uploads/2015/09/Manual-deBiosseguran\%C3\%A7a-do-Curso-de-Enfermagem-Finalizado-3.pdf.

18. Ministério do Trabalho e Emprego (BR). Portaria n. 485, de 11 de novembro de 2008. Aprova a Norma Regulamentadora $n^{\circ} 32$. Segurança e Saúde no Trabalho em Estabelecimentos de Saúde. Diário Oficial da União, [Internet]. $11 \mathrm{dez} 2008$ [acesso em 04 out 2017]. Disponível em: http://sbbq.iq.usp.br/arquivos/ seguranca/portaria485.pdf.

19. Santos LT dos, Rocha FLR, Marziale MHP. Agulhas com dispositivos de segurança e a prevenção de acidentes: revisão integrativa. Rev. bras. enferm. [Internet]. 2018 [acesso em 12 fev 2019]; 71(6). Disponível em: http://dx.doi.org/10.1590/0034-7167-2017-0719.

20. Bouchoucha SL, Moore KA. Factors Influencing Adherence to Standard Precautions Scale: a psychometric validation. Nurs Health Sci [Internet]. 2018 [acesso em 12 fev 2019]; 21(2). Disponível em: https://doi.org/10.1111/nhs.12578.

Recebido: 06/05/2019

Finalizado: 13/02/2020

\author{
Autor Correspondente: \\ Edvan Adalberto Dias dos Passos \\ Universidade de São Paulo \\ R. Monte Alegre, 520 - 14051-260 - Ribeirão Preto, SP, Brasil \\ E-mail: edvan.passos@usp.br
}

Contribuição dos autores:

Elaboração e revisão crítica do conteúdo intelectual do estudo - EADP

Responsável por todos os aspectos do estudo, assegurando as questões de precisão ou integridade de qualquer parte do estudo - MHPM 